\title{
A Practical Strain Sensor Based on Ecoflex/Overlapping Graphene/ Ecoflex Sandwich Structures for Vocal Fold Vibration and Body Motion Monitoring
}

\author{
Yanyan Fan ${ }^{1,2}$, Hongbin Zhao ${ }^{1 *}$, Yifan Yang ${ }^{3,4}$, Yi Yang ${ }^{3,4}$, Tianling Ren ${ }^{3,4 *}$ and Hailing $\mathrm{Tu}^{1 *}$ \\ ${ }^{1}$ Key Laboratory of Advanced Materials for Smart Sensing, GRINM Group Co., Ltd., Beijing, China, ${ }^{2}$ China Academy of Space \\ Technology, Beijing, China, ${ }^{3}$ Institute of Microelectronics, Tsinghua University, Beijing, China, ${ }^{4}$ Beijing National Research Center \\ for Information Science and Technology (BNRist), Tsinghua University, Beijing, China
}

OPEN ACCESS

Edited by:

SungWoo Nam,

University of Illinois at UrbanaChampaign, United States

Reviewed by:

Jin Myung Kim,

University of Illinois at Urbana-

Champaign, United States

Juyoung Leem,

Stanford University, United States

*Correspondence:

Hongbin Zhao

zhaohongbin@grinm.com

Tianling Ren

rent/@tsinghua.edu.cn

Hailing Tu

tuhl@grinm.com

Specialty section: This article was submitted to

Sensor Devices,

a section of the journal

Frontiers in Sensors

Received: 17 November 2021

Accepted: 10 December 2021

Published: 06 January 2022

Citation:

Fan $Y$, Zhao $H$, Yang $Y$, Yang $Y$, Ren $T$ and Tu H (2022) A Practical Strain

Sensor Based on Ecoflex/Overlapping Graphene/Ecoflex Sandwich Structures for Vocal Fold Vibration and

Body Motion Monitoring.

Front. Sens. 2:815209.

doi: 10.3389/fsens.2021.815209
Graphene-based stretchable and flexible strain sensors are one of the promising "bridges" to the biomedical realm. However, enhancing graphene-based wearable strain sensors to meet the demand of high sensitivity, broad sensing range, and recoverable structure deformation simultaneously is still a great challenge. In this work, through structural design, we fabricated a simple Ecoflex/Overlapping Graphene/Ecoflex (EOGE) strain sensor by encapsulating a graphene sensing element on polymer Ecoflex substrates using a dropcasting method. The EOGE strain sensor can detect stretching with high sensitivity, a maximum gauge factor of 715 with a wide strain range up to $57 \%$, and adequate reliability and stability over 1,000 cycles for stretching. Moreover, the EOGE strain sensor shows recoverable structure deformation, and the sensor has a steady response in the frequency disturbance test. The good property of the strain sensor is attributed to the resistance variation induced by the overlap and crack structure of graphene by structural design. The vibrations caused by sound and various body movements have been thoroughly detected, which exhibited that the EOGE strain sensor is a promising candidate for wearable biomedical electronic applications.

Keywords: overlapping graphene, strain sensor, bioelectric, health monitor, wearable, stretchable, highly sensitive

\section{INTRODUCTION}

In recent years, flexible and stretchable sensors have become one of the most widely studied fields for their potential application in next-generation bioelectronics, including electronic skin, in vitro diagnostics, and human physiological motion detections (Ahn and Hong, 2014; Zhang et al., 2015; Park C. W. et al., 2016; Song et al., 2016; Han et al., 2018; Liu R. et al., 2018; Tian et al., 2018; Wang S. et al., 2018; Green Marques et al., 2019; Lee et al., 2019; Li et al., 2019). Owing to the ability to transduce complex mechanical deformations to electrical feedback, wearable strain sensors received a great deal of attention (Pang et al., 2012; Li et al., 2015; Qin et al., 2015; Roh et al., 2015; Park S.J. et al., 2016). Generally, the performance of the wearable strain sensors is evaluated by the flexibility, stretchability, sensitivity, and durability (Park S.-J. et al., 2016). Although traditional strain sensors based on metals and semiconductors have been well developed, their limited sensing range $(<5 \%)$ and rigidity hinder their applications in wearable electronics (Liu Q. et al., 2016; Liu Y. et al., 2016; 
Gao et al., 2018). Human activity monitoring includes not only subtle human physiological signals such as wrist pulse and heartbeat but also large-scale motions such as movements of the finger, elbow, and knee. For example, the movement of elbow bending can generate strains over 50\%, which far exceeds the sensing range of traditional technology (Li et al., 2017). Therefore, for practical applications, wearable strain sensors are needed to fulfill not only large stretchability for high strain but also high sensitivity to small strain.

Currently, various approaches have been developed to enhance the performance of flexible and stretchable strain sensors based on different mechanisms, including piezoresistance, capacitance, and piezoelectricity (Cai et al., 2013; Park et al., 2014; Shin et al., 2014; Han et al., 2018). Particularly, piezoresistance-type sensors have more advantages due to their simple device structures and relatively easy readout systems (Liao et al., 2017). A number of nanomaterials, such as nanoparticles (Park et al., 2012; Lee et al., 2014), nanowires (Gong et al., 2014), nanotubes (Park S.-J. et al., 2016; Hao et al., 2018), and two-dimensional materials, have been employed to prepare piezoresistance-type strain sensors with the desired performance. Among them, graphene with extraordinary electrical and mechanical properties has been considered one of the most promising candidates to build wearable strain sensors (Chen et al., 2011; Bae et al., 2013). Recently, graphene-based piezoresistive strain sensors have been widely studied (Kim et al., 2009; Liu Y. et al., 2016; Wang Y. et al., 2018; Yan et al., 2018). Wang et al. showed a graphene-based strain sensor, which had an ultrahigh sensitivity (1,000 under $2-6 \%$ strains) but limited strain range up to $7 \%$ (Wang et al., 2014). Yan et al. reported a highly stretchable strain sensor based on graphene nanocellulose nanopaper which could be stretched over $100 \%$ strain, but the sensitivity of the strain sensor was only 7.1 (Yan et al., 2014). A broad sensing range requires connections between the sensing layers when subjected to large strains, while the achievement of high sensitivity needs the generation of microstructure changes under small deformation. Recently, a graphene foam-based strain sensor was reported for its high sensitivity and a good stretchability of 95\% (Chen et al., 2011). However, the fabrication method involved the high-cost chemical vapor deposition (CVD) technology and complicated procedures. It remains a significant demand to develop facile and low-cost techniques to fabricate high-performance graphene strain sensors with a wide detective range.

In our previous work, reduced graphene oxide (rGO) and elastomer were used in the fabrication of wearable strain sensors (Wang et al., 2016; Tao et al., 2017), which displayed relatively large stretchability and high sensitivity properties due to the highdensity cracks. Here, we designed a highly flexible, stretchable, sensitive, and reliable EOGE sandwich structure with low resistance overlapping graphene as the sensitive material and elastic polymer Ecoflex as the flexible matrix. The sensing characteristics of the EOGE strain sensor including stretch response to dynamically and statically applied strains, stretchability, and durability performance have been investigated, which exhibits a relatively broad sensing range (up to 57\%), moderate sensitivity (GF $=100-715$ ), and good cycling stability ( 1,000 stretching/releasing cycles). We also detected the retention property, working frequency, and vibrations caused by wrist pulse, real-time breathing, sound and facial expression, movements by the knee and elbow bending, etc. Our current work showed the simply fabricated, low-cost, and high-performance sensing devices have potential applications in wearable bioelectronics.

\section{EXPERIMENTAL SECTION}

\section{Fabrication of the Strain Sensor}

The EOGE sandwich structure strain sensor was prepared by using the following procedures, as illustrated in Figure 1. First, the prepolymer and cross-linker of an Ecoflex silicone elastomer (Ecoflex 0,030; Smooth-On, Inc., United States) were mixed together in a mass ratio of $1: 1$, mechanically stirred thoroughly, poured onto a cleaned glass slide, and then cured at room temperature for $2 \mathrm{~h}$. The Ecoflex was selected as the flexible matrix material due to its good stretchability, durability, and biocompatibility (Amjadi et al., 2015). The commercially available polyimide (PI) tape (with a rectangular pattern size of roughly $1 \times 4 \mathrm{~cm}$ ) was attached on the cured Ecoflex substrate as a shadow mask. Graphene ink (a sheet diameter of 1-3 $\mu \mathrm{m}$, XFNANO Materials Tech CO. Ltd., Nanjing, China) was subsequently drop-cast onto the patterned Ecoflex and kept in the fuming cupboard in order to form a film. Next, the PI tape was stripped away and copper wires were attached to the ends of the graphene film that served as the electrodes. Silver paste was used to eliminate the contact resistance by drying under an infrared lamp at $60^{\circ} \mathrm{C}$ for $12 \mathrm{~h}$. Then another liquid Ecoflex layer was cast on top of the patterned graphene film to encapsulate the whole structure. Finally, the complete sample was cured at room temperature for another $2 \mathrm{~h}$. The strategy of structural design of the EOGE strain sensor has the advantages of a simple preparation process, mild conditions, and low cost and is particularly suitable to be used for mass production.

\section{Characterization and Performance Testing}

The morphologies and structures of the graphene film were characterized by a field emission scanning electron microscope (FE-SEM, Zeiss Merlin, and Germany). The film thickness was analyzed using a 3D optical surface metrology system microscope ((Leica DCM8, Leica, Germany). Raman spectroscopy was employed using a laser of $532 \mathrm{~nm}$ wavelength (HORIBA Inc.). The loading of tensile strain was performed with a universal testing machine (SHIMADZU AGS-X), and the electrical signals of the strain sensor were recorded by a digital multimeter (GOGOL DM3068). The sensor was mounted on the wrist, elbow, knee, neck, cheek, throat, and abdomen to monitor human body motions in real time.

\section{RESULTS AND DISCUSSION}

The morphology of the overlapped graphene film was clearly observed in the top view of SEM images (Figure 2A). The images 


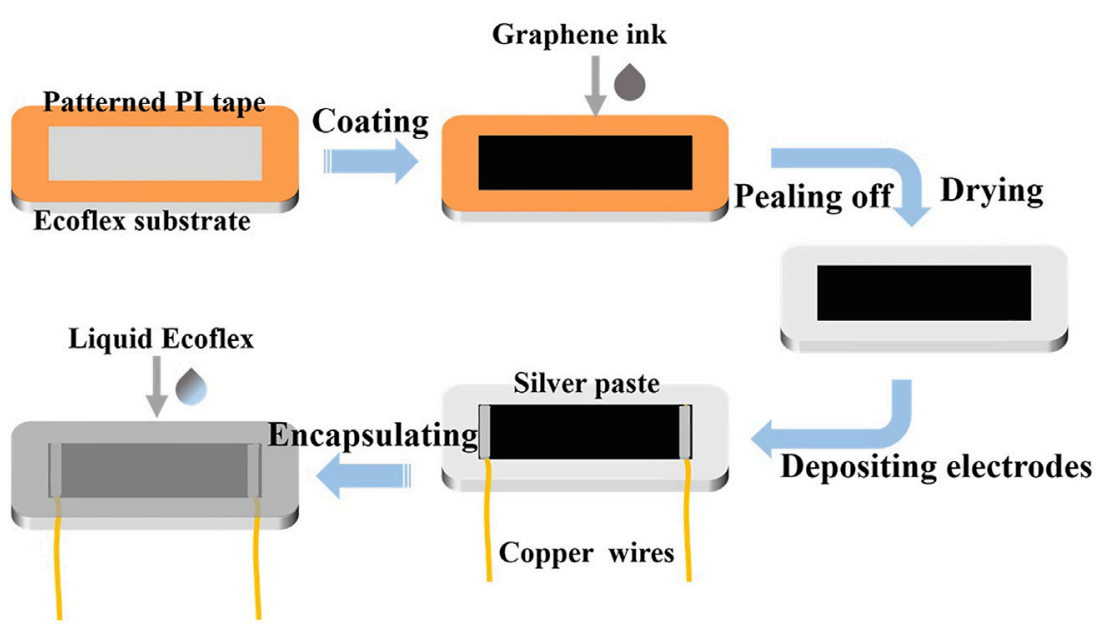

FIGURE 1 | Schematic illustration for the preparation of the flexible graphene strain sensor.
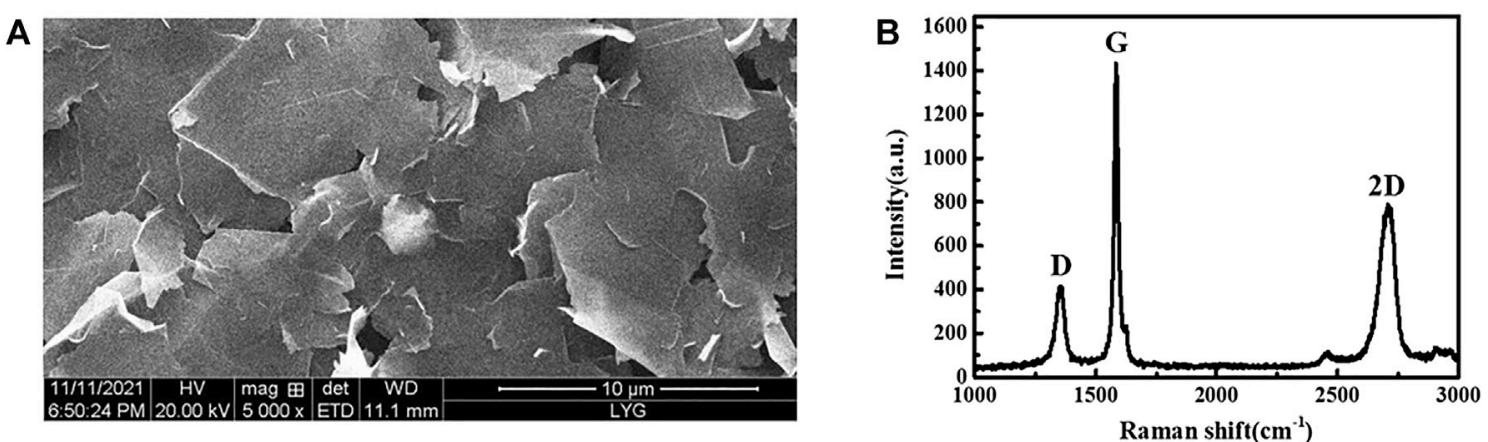

FIGURE 2 | Characterization of the overlapped graphene film: (A) SEM image of the overlapped graphene film without strain; (B) Raman spectrum of the graphene film.

illustrate that the compactly constructed and closely stacked adjacent graphene flakes are bounded and stacked via $\pi-\pi$ interactions. Therefore, once the graphene flakes move along with the substrate during stretching, the strain deformation will reduce the overlap area between adjacent flakes, which eventually leads to the detachment of the flakes and microcracks in the sensing layer. The Raman spectra of the graphene film, illustrated in Figure 2B, show three typical bands: a D-band at $1,352 \mathrm{~cm}^{-1}$, a G-band at $1,581 \mathrm{~cm}^{-1}$, and a $2 \mathrm{D}$-band at $2,707 \mathrm{~cm}^{-1}$. The D-band is considered to attribute to the structural defects related to the partially disordered structures or the attachment of functional groups on the graphitic domains (Kato et al., 2019). The G-band is associated with the $\mathrm{sp}^{2}$ carbon hybridization in the graphene film. The ratio of peak intensities of the $\mathrm{D}$ band and $\mathrm{G}$ band $\left(\mathrm{I}_{\mathrm{D}} /\right.$ $\mathrm{I}_{\mathrm{G}}$ ) was 0.28 , indicating a low density of defects in the graphene film (Wu et al., 2018; Kato et al., 2019). The 2D band has also been observed, whose intensity and symmetry are the fingerprint signals of graphene. With an $\mathrm{I}_{2 \mathrm{D}} / \mathrm{I}_{\mathrm{G}}$ ratio of 0.55 , the film is expected to be multilayered (Bayle et al., 2018).

In order to fully assess the strain sensing properties of the sensor, real-time resistance with respect to strain was investigated in detail. The electrical response is defined as the variation of relative resistance, namely, $\Delta R / R_{0}=\left(R-R_{0}\right) / R_{0}$, where $R_{0}$ and $R$ correspond to the resistance of the sensor without and with applied strain, respectively. The strain is defined as $\varepsilon=\left(\mathrm{L}-\mathrm{L}_{0}\right) /$ $\mathrm{L}_{0}$, where $\mathrm{L}_{0}$ and $\mathrm{L}$ correspond to the length of the sensor without and with applied strain, respectively.

To evaluate the strain sensing range and sensitivity of the EOGE strain sensor, a simple strain test has been carried out. As shown in Figure $\mathbf{3 A}$, the electrical response exponentially increased from original 0 to ultimate 407.6 when the applied strain is increased from 0 to $57 \%$. Gauge factor (GF) is a characteristic parameter representing the sensitivity of the strain sensor and is defined as follows:

$$
\mathrm{GF}=\left(\Delta \mathrm{R} / \mathrm{R}_{\mathrm{o}}\right) / \varepsilon
$$

where $\Delta R$ is the variation of relative resistance, $R_{0}$ is the original resistance of the sensor, and $\varepsilon$ stands for the applied strain. The gradient of the curve in Figure $3 \mathrm{~A}$ reflects the GF of the sensor, and the GF values generally increase with the increase in the strain (see Figure 3B). When the applied strain is increased up to $57 \%$, the GF values reach 

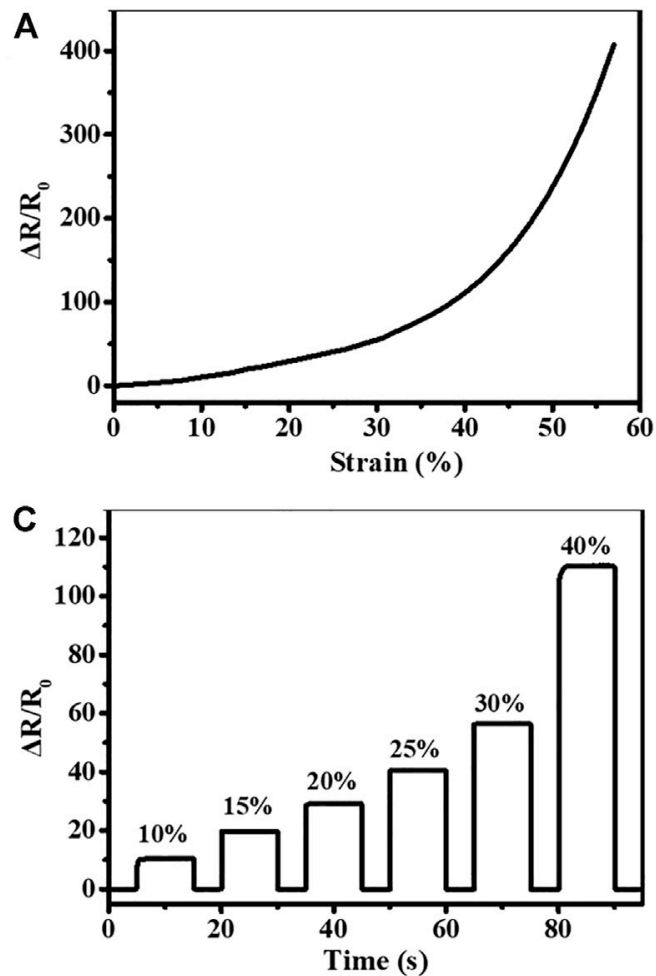

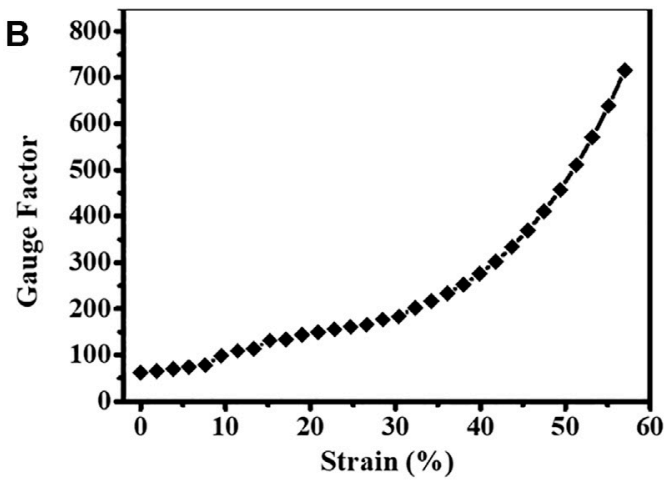

D

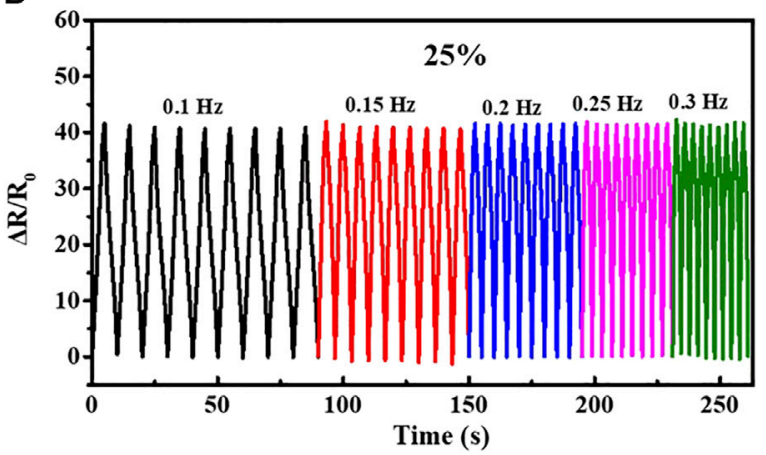

FIGURE 3 | Electrical properties of the graphene strain sensor. (A) Relative resistance vibration of the sensor during stretching; (B) the gauge factor of the sensor versus strain; (C) relative resistance vibration-time curve of the strain sensor under 10, 15, 20, 25, 30, and 40\% strains with static-hold periods; (D) relative resistance vibration under cyclic stretching/releasing at frequencies of $0.1,0.15,0.2,0.25$, and $0.3 \mathrm{~Hz}$.

TABLE 1 | Comparison of sensing performance between other previously reported sensors and the sensor in this work.

\section{Material}

AuNWs/Ecoflex

Carbon nanotubes/PDMS

Graphene-ionic conductor/Ecoflex

rGO/Ecoflex

Graphene nanopaper

Graphene film/elastic tape

Graphene foam/PDMS

Graphene/Ecoflex

rGO/PDMS

rGO/Ecoflex

Graphene ink/Ecoflex

\section{Maximum stretchability (\%)}

350

100

300

400

100

82

70

50

7.5

35

57
Maximum GF

\section{9}

62

25.2

31.6

7.1

150

29

522.6

402.5

457

715
Reference

Gong et al. (2015) Roh et al. (2015)

Liu C. et al. (2018) Xu et al. (2018)

Yan et al. (2014)

Liu Q. et al. (2016)

Jeong et al. (2015)

Amjadi et al. (2016)

Wang et al. (2016)

Tao et al. (2017)

This work to a maximum of 715 . The strain sensor exhibits a desirable integration of high gauge factor and wide sensing range. The maximum workable strain range and maximum gauge factor of our EOGE strain sensor and other elastomer-based strain sensors in literatures are summarized in Table 1. We could find that previously reported strain sensors, including graphene-based materials, usually have narrow sensing ranges $(<10 \%)$ and/or low sensitivities (Yan et al., 2014; Gong et al., 2015; Jeong et al., 2015; Roh et al., 2015; Amjadi et al., 2016; Liu Q. et al., 2016; Wang et al., 2016; Tao et al., 2017; Liu C. et al., 2018; Xu et al., 2018).
The static-hold strains during the cycles of the loading-unloading process have been measured to demonstrate the retention property of our EOGE strain sensor. The electrical response of the strain sensor has systematically been recorded by applying cyclic strains from 0 to maximal values of $10,15,20,25,30$, and $40 \%$, and the corresponding curve is plotted in Figure 3C. In the first cycle, the strain was increased to maximal values and held for $10 \mathrm{~s}$; then it was released to $0 \%$ and held again for another $5 \mathrm{~s}$. The electrical response maintains a distinct plateau regime until the applied strain is released, indicating well-behaved stability at various 

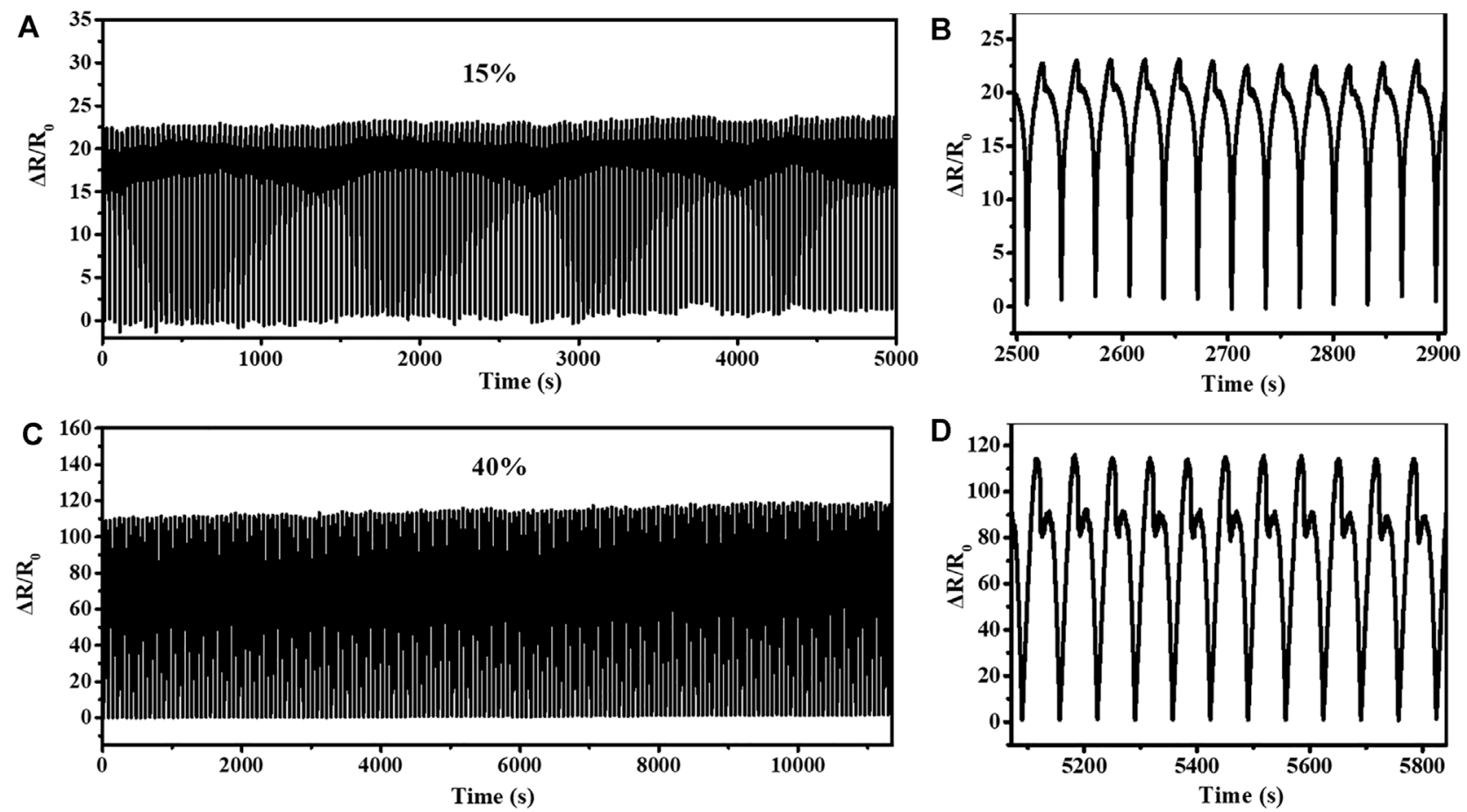

FIGURE 4 | (A) Multicycle operation of the strain sensor under 15\% strain, (B) the magnified cycles of the stretching test results, (C) multicycle operation of the strain sensor under $40 \%$ strain, and (D) the magnified cycles of the stretching test results.

strain stages. In addition, the electrical responses upon stretching to maximum strains of $10,15,20,25,30$, and $40 \%$ were measured to be about $10.34,19.71,29.34,40.59,54.82$, and 111.05, respectively, and the results are consistent with those in Figure 3A.

Furthermore, working frequency is considered to be a major characteristic of the strain sensor needed (Boland et al., 2014). The cyclic stretching-releasing strain at a frequency range from 0.1 to $0.3 \mathrm{~Hz}$ was investigated. As shown in Figure 3D, the slope of the response curve is relevant with the frequency for each cycle, and it follows the rule that larger working frequency corresponds to a steeper slope. The trend in the output electrical response can keep pace with that of the input applied strain. The EOGE strain sensor exhibits little rate dependence to the working frequency within the range of applied frequency, indicating the fast and reliable response of the sensor.

The reliability and mechanical durability are used to evaluate the consistency of the results obtained from the repeated measurements of each sample in a short time under the same measurement condition. The reliability and mechanical durability of our strain sensor have been evaluated by applying strains of 15 and $40 \%$ to the strain sensor for 1,000 repeated stretching/releasing cycles, and the results are shown in Figures 4A,C. During the cycling tests, the cyclic change in relative resistance variations of around 20 and 111 with 15 and $40 \%$ stretch is observed. When the applied strain is released, the response can be restored to its original state. Figures 4B,D represent several random stretching/releasing cycles extracted from the multi-cycle tests of Figures 4A,C. Remarkably, no significant decrease in the response has been observed over the process of repeated stretching/releasing cycles, demonstrating that the strain sensor is mechanically robust and reliable in the multi-cycle tests, which is of critical importance for applications.

In order to reveal the sensing mechanism of the strain sensor, the microstructures of the strain sensor without upper side Ecoflex before and after being stretched are investigated. As shown in Figure $\mathbf{5 A}$, before stretching, the graphene film exhibits a rough but continuous morphology, without signs of separating or splitting. When suffered a small range of strains, the graphene film began to break (see Figure 5B). Several cracks appeared on the surface of the graphene film, resulting in the increase in resistance of the conductive film. As the strain was increased gradually, more cracks on the graphene film appeared and adjacent cracks connected to form a bigger crack, resulting in a larger resistance change, which has also been observed in the previous studies (Yan et al., 2014; Liu Q. et al., 2016; Liu Y. et al., 2016; Xu et al., 2018).

During the stretching process, the resistance variation of the sensing layer arose due to the change in the contact conditions. This primarily includes two aspects: first, the widening of cracks caused by the break of contacts upon stretching and second, the contact area and spacing variations such as the reconnection and overlapping of cracks (Li et al., 2012; Wang et al., 2014). Hence, a simplified model based on the changing of graphene flakes in the sensing layer with and without strain from the top view and side view is presented in Figures 5D,E. According to this model, when a strain is applied, some graphene flakes are separated and a 
A
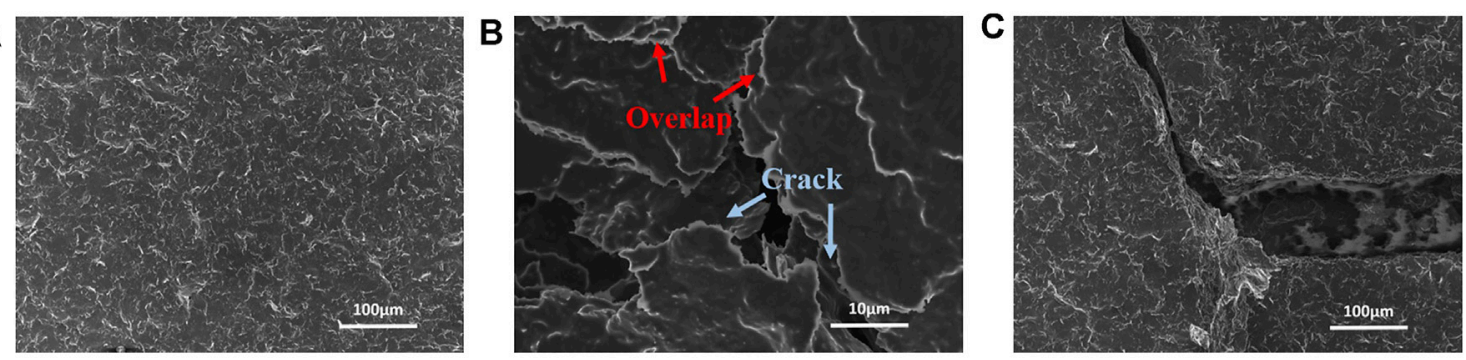

D

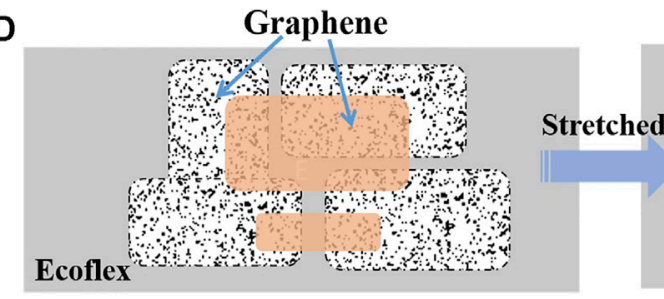

Overlap

Crack

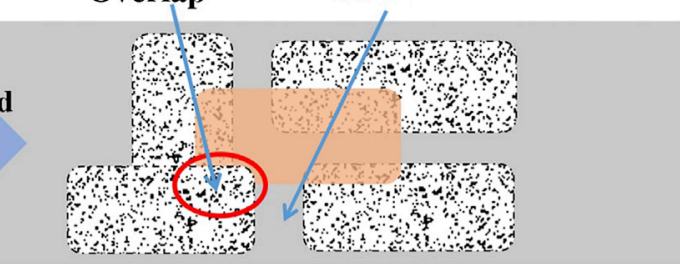

Top

view

E

e

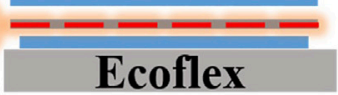

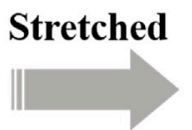

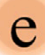

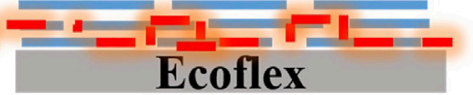

FIGURE 5 | SEM images of the flexible graphene strain sensor (A) without strain, and (B, C) under a stretched state; schematic structure of the strain sensor with and without strain from (D) the top view and (E) the side view.

number of cracks generated, which lead to a remarkable increase in the graphene-sensing layer along the stretching direction. Cracks appear and become increasingly large with the extension of the strain.

From the side view, for the pristine state, the stacked layers are in contact with each other, creating perfect conductive pathways. When strain is applied, it can generate separation between the conductive layers perpendicular to the stretching direction. This separation attributes to the decrease in conductive pathways, leading to a slight increase in resistance.

For further details, to analyze the mechanism concretely, we employ the finite-element method and set a model of a network of randomly positioned graphene flakes to analyze the strain effect. Resistance is calculated by Ohm's law, $\mathrm{R}=\mathrm{V} / \mathrm{I}$ under the fixed current. In the graphene flakes network, the voltage drop is not uniform at current direction but depends on the electrical conductivity distribution. Ohm's law could be rewritten as follows in two dimensions:

$$
-\nabla(\sigma \nabla \varphi)=j
$$

In the previous equation, $\sigma$ is the electrical conductivity; $j$ is the current density flux into the plane, which is 0 in the area of strain sensor; and $\varphi$ is the electronic potential. The potential distribution is computed by the finite-element method under the electrical conductivity distribution and fixed current. The strain effect is analyzed by modeling the percolation of current through a network of randomly positioned circular flakes with a uniform size distribution. As shown in Figure 6A, the electrical conductivity distribution depends on the distribution of graphene flakes, and it is higher in the overlap area of multi-layer graphene flakes. The decrease in the overlap of graphene flakes changes the electrical conductivity of the overlap area, which means the whole resistance increases since the overlap area decreases when the device is stretched. A few sub-circuits are even disconnected as the graphene flakes move to non-overlapping positions. All these effects increase the resistance of the device as the experimental result. When stretched, the crack and overlap generate as illustrated. Figure 6B shows the simulated curve of the strain versus GF, where the simulated result well matched with the experimental data.

Since our strain sensor has a good comprehensive performance including biocompatibility, high flexibility, stretchability, and sensitivity, it was employed to realize realtime detection of multi-scale human motion as a wearable sensing device, including human physiological signals and vigorous joint movement. In terms of human physiological signals, the sensor responds to pulse, breath, phonation, and expression. Because of the flexibility of the sensor, it was simply attached to the curved parts of the human body using medical tape to facilitate various biosignal measurements. The pulse is a useful and valuable physiological indicator for non-invasive medical diagnosis; thus, it is of great importance to perceive pulse signals by strain sensors. Figures 7A,B show a case in which the sensor is tightly attached to the skin over the artery in a human wrist to monitor the successive output subtle pulse signal. The obtained pulse rate of the tester under normal conditions is 73 times $\mathrm{min}^{-1}$, which is within the normal range of a healthy adult. Each signal wave exhibits a regular and repeatable response curve. Important 

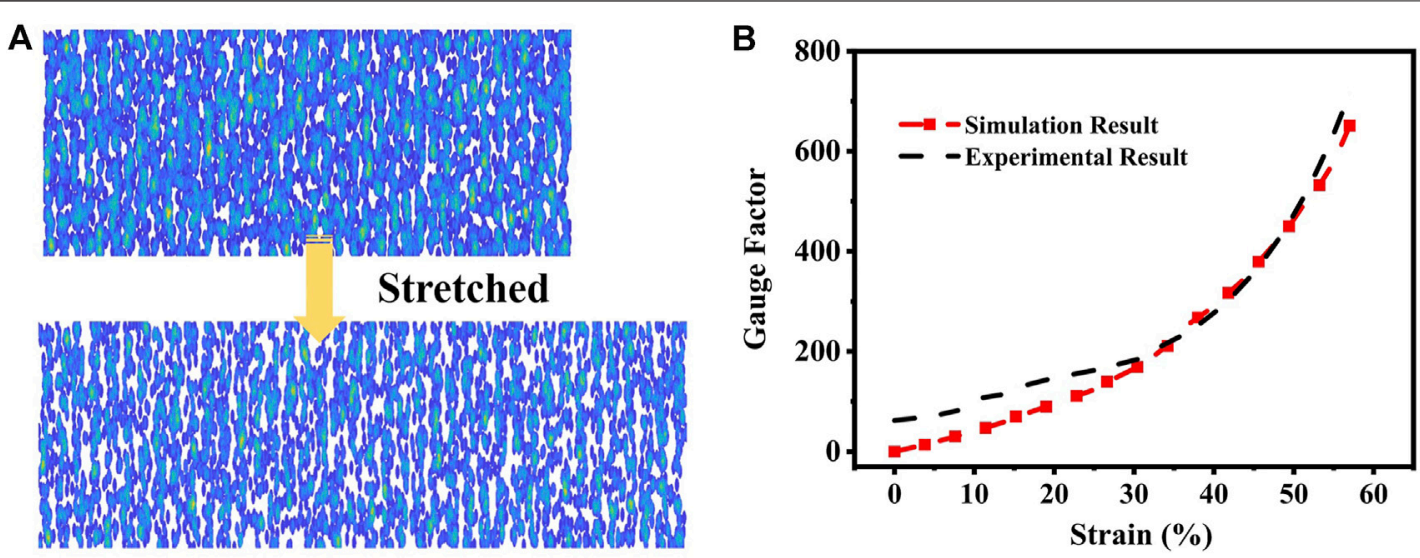

FIGURE 6 | (A) Randomly positioned graphene scales model without and with strain; (B) the simulation result of GF versus strain (the red dash dotted line) matches well with the calculated result (the black line).

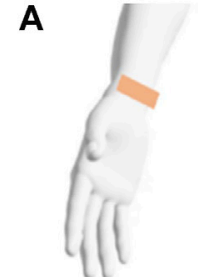

F

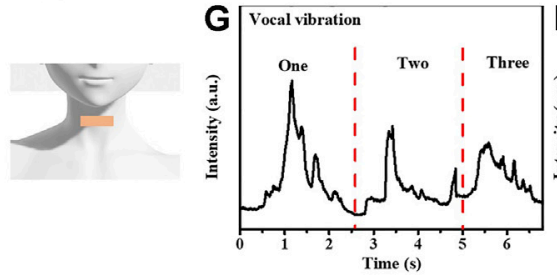

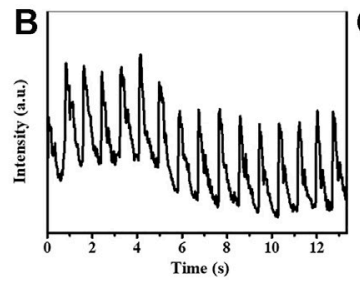
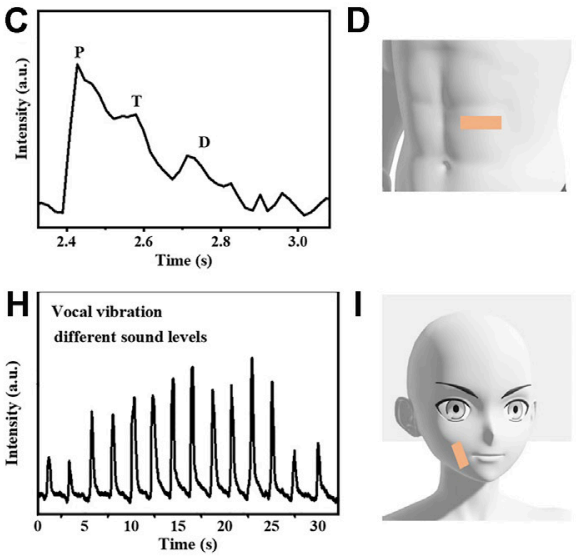

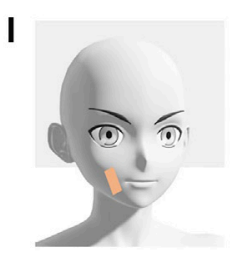

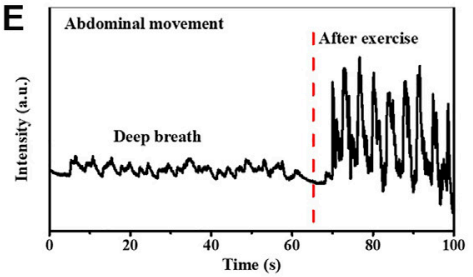

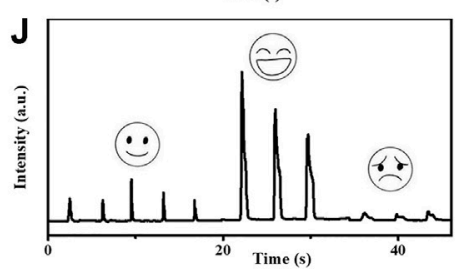

FIGURE 7 | Applications of the flexible graphene strain sensor for detecting various subtle human motions: (A, D, F, and I). Schematic illustrations of the sensor attached to different parts for detecting subtle human motions; (B) The electrical response induced by the wrist pulse; (C) Magnified image of a single pulse, revealing typical characteristics of the pulse; (E) Detecting of the respiration rate with different breath modes; (G) Electrical response towards different words pronounced by the tester; (H) Electrical response towards different sound levels; $(\mathbf{J})$ Electrical response of different facial expressions.

information about the arterial wall could be obtained from the arterial waveform, including the stiffness of the aortic system as well as the speed and pressure of the pulse. In Figure 7C, which is a magnified view of a single pulse, three distinguishable characteristic peaks can be observed, associating with the percussion wave (P-wave), tidal wave ( $\mathrm{T}$-wave), and diastolic wave (D-wave), respectively, which can provide meaningful biomedical information for health-care diagnosis (Liu C. et al., 2018). Therefore, the strain sensor is suitable as a non-invasive diagnostic device for identifying tiny mechanical signals.

Figures 7D,E show a real-time trace revealing the repeated abdominal movement of the tester while breathing. The amplitude and frequency of breathing are monitored simultaneously by a waist belt integrated with the strain sensor. Briefly, two breathing modes, namely, deep breathing and heavy breathing after exercise, can be identified from the electrical response time curve. The upward and downward slopes of the response signal pattern are correlated with stretch during inhalation and release during exhalation, respectively, and the shape of the electrical response is associated with the different breathing modes. According to the obtained results, for deep breathing, the intensity of the relative resistance change is comparatively low, whereas heavy breathing after exercise exhibits high amplitude of the relative resistance change. Apart from tracing the amplitude of breathing, the breathing frequency of the tester can also be easily calculated based on the peak numbers at a certain time. The deep breathing rate of the tester has been calculated approximately to be 15 times per minute, whereas it has been increased to 24 times per minute after exercise. The strain sensor provides an efficient and simple method for long-term respiration monitoring. 

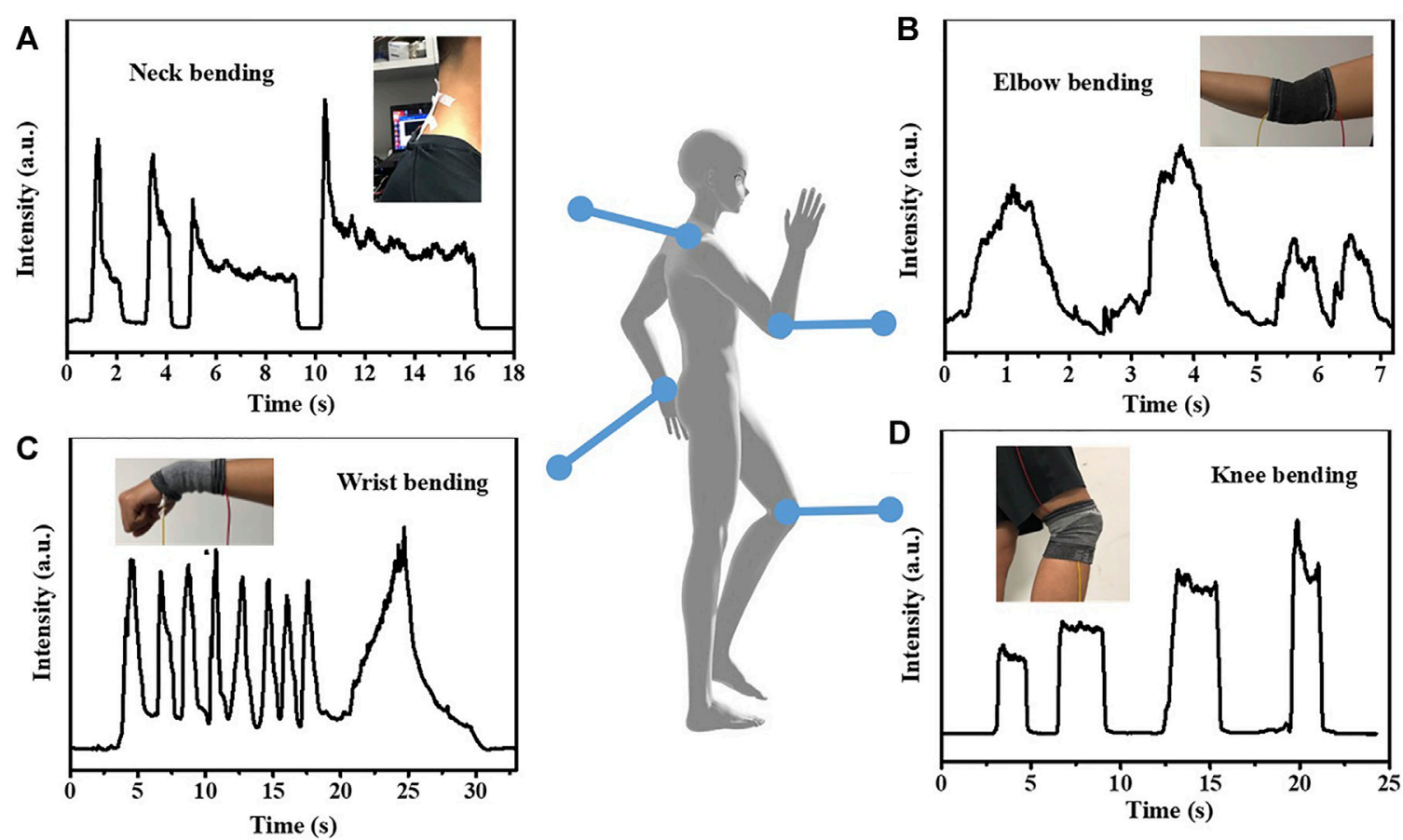

FIGURE 8 | Applications of the flexible graphene strain sensor for detecting various large human motions. Corresponding electrical response to (A) neck bending, (B) elbow bending, (C) wrist bending, and (D) knee bending.

Furthermore, the strain sensor is attached to the throat of a person to monitor the delicate epidermis/muscle movements of phonation of words (see Figure 7F). The sensor shows distinguishable characteristic sensing signal patterns as the tester spoke several different words, including "one," "two," and "three" (Figure 7G), since each word resulted from distinct motion of the vocal chords. The response has also been observed to be associated with the volume of pronunciation, as revealed in Figure $7 \mathbf{H}$. Good phonation recognition represents this sensor has promising applications in human/machine interaction and phonation rehabilitation exercise.

Besides, detection of facial expression has great importance in monitoring the state of mind through emotions. Thus, the strain sensor is attached to the cheek of the tester to effectively monitor the muscles around the mouth of the tester (see Figure 7I). The strain sensor is sensitive to the small movements of the muscle and shows a clear response with relatively repeatable signal patterns in different cases (see Figure 7J), whereas differences in the degree of muscle movements of the mouth slightly alter the response of the strain sensor.

Aside from detection for subtle human physiological signals, the strain sensor is also used to monitor diverse relatively large body motions of different human body parts. The sensor can be used to monitor the habits that cause deformity, for example, in the spinal cord. It can be attached on the neck of the tester to monitor the sitting position and the response against time with varying bending angles as the tester nods periodically (see
Figure 8A). In this way, the sensor may be useful for warning for poor postures and preventing slouching.

The sensing signals of large body joints, including elbow, wrist, and knee bending, were studied and are exhibited in Figures 8B-D. The increase in the bending angle results in larger electrical response signals. Figure $\mathbf{8 B}$ demonstrates the ability of the sensor to detect elbow bending. The strain sensor was integrated with an elbow wrap and was then placed at the arm and located at the outside of the elbow. When the elbow was bent at a certain angle, the electrical response increased abruptly. In bending cycles, the strain sensor showed a real-time response to the elbow motion and favorable reproducibility. The sensor produced signals with different intensities since larger bending motions result in greater increment in the relative resistance change. When the sensor was applied to the wrist, its bending motions were also easily monitored (see Figure 8C). Once the wrist was bent, the deformation of the wrist can be adopted by the strain sensor. The sensor responds to the motion of the finger bending fast and recovers to its pristine state at each bending-releasing cycle, which exhibits a repeatable pattern. Different wrist bending rates can be easily reflected by the variations in the relative resistance of the sensor. To monitor knee bending (see Figure 8D), the sensor was simply mounted onto the knee pads worn on the knee by two laces and the bending of knees can be easily detected by monitoring the relative resistance variation during one-legged seated calf raises. The resistance variation increases in a stepwise manner in real time as the bending angle of knees increases. Therefore, it can 
be used to reflect movement state of the person and to develop applications for wearable electronics.

\section{CONCLUSION}

In this work, we developed a facile and low-cost method to fabricate a highly stretchable and sensitive strain sensor using the patterned Ecoflex polymer elastic substrate and the graphene ink sensing material. The fabricate process sensing performance and working mechanism of the strain sensor have been elaborated. The strain sensor exhibits high performance with a relatively good stretchability of $57 \%$ strain with a GF of 715 , which are higher than those of the conventional strain sensors, and manifests itself in detecting both subtle and large strains induced by vocal fold vibrations and body motions. Moreover, it also showed good durability over 1,000 cycles (for 15 and $40 \%$ strain, respectively) and stability for various applied static strains and frequencies. A model has been established to illuminate the sensor deformation behavior during the stretching and releasing processes. The strain changed the overlap area of the contacting and induced crack to the film, leading to the increase in the resistance. Since the strain release can cause the reconstruction of the contacting area and film, the resistance of the sensing layer decreases. We demonstrate that the sensor can be directly attached to the human skin and generated distinguishable resistance characteristics corresponding to diverse human activity

\section{REFERENCES}

Ahn, J.-H., and Hong, B. H. (2014). Graphene for Displays that bend. Nat. Nanotech 9, 737-738. doi:10.1038/nnano.2014.226

Amjadi, M., Turan, M., Clementson, C. P., and Sitti, M. (2016). Parallel Microcracks-Based Ultrasensitive and Highly Stretchable Strain Sensors. ACS Appl. Mater. Inter. 8, 5618-5626. doi:10.1021/acsami.5b12588

Amjadi, M., Yoon, Y. J., and Park, I. (2015). Ultra-stretchable and Skin-Mountable Strain Sensors Using Carbon Nanotubes-Ecoflex Nanocomposites. Nanotechnology 26, 375501. doi:10.1088/0957-4484/26/37/375501

Bae, S.-H., Lee, Y., Sharma, B. K., Lee, H.-J., Kim, J.-H., and Ahn, J.-H. (2013). Graphene-based Transparent Strain Sensor. Carbon 51, 236-242. doi:10.1016/ j.carbon.2012.08.048

Bayle, M., Reckinger, N., Felten, A., Landois, P., Lancry, O., Dutertre, B., et al. (2018). Determining the Number of Layers in Few-Layer Graphene by Combining Raman Spectroscopy and Optical Contrast. J. Raman Spectrosc. 49, 36-45. doi:10.1002/jrs.5279

Boland, C. S., Khan, U., Backes, C., O’Neill, A., McCauley, J., Duane, S., et al. (2014). Sensitive, High-Strain, High-Rate Bodily Motion Sensors Based on Graphene-Rubber Composites. ACS Nano 8, 8819-8830. doi:10.1021/ nn503454h

Cai, L., Song, L., Luan, P., Zhang, Q., Zhang, N., Gao, Q., et al. (2013). Superstretchable, Transparent Carbon Nanotube-Based Capacitive Strain Sensors for Human Motion Detection. Sci. Rep. 3, 3048. doi:10.1038/srep03048

Chen, Z., Ren, W., Gao, L., Liu, B., Pei, S., and Cheng, H.-M. (2011). Threedimensional Flexible and Conductive Interconnected Graphene Networks Grown by Chemical Vapour Deposition. Nat. Mater 10, 424-428. doi:10.1038/nmat3001

Gao, J., Wang, X., Zhai, W., Liu, H., Zheng, G., Dai, K., et al. (2018). Ultrastretchable Multilayered Fiber with a Hollow-Monolith Structure for signals, including subtle physiological activities (e.g., wrist pulse and respiration) and large-scale motions (e.g., knee bending and wrist bending). Based on these demonstrations, our study provides a practical and general strategy of fabricating a highly stretchable and sensitive strain sensor and develops promising applications in advanced wearable electronics, such as human motion detection and health monitoring.

\section{DATA AVAILABILITY STATEMENT}

The original contributions presented in the study are included in the article/Supplementary Material; further inquiries can be directed to the corresponding authors.

\section{AUTHOR CONTRIBUTIONS}

YF and TR designed the experiments. YF and YY performed the experiments. YF and YY analyzed the data and wrote the article. $\mathrm{HZ}$ and $\mathrm{HT}$ reviewed, revised, and edited the article.

\section{FUNDING}

This work was supported by the National Key R\&D Program (2016YFA0200400) and National Natural Science Foundation (51861145202, 61574083, 61434001, and 61874137).

High-Performance Strain Sensor. ACS Appl. Mater. Inter. 10, 34592-34603. doi:10.1021/acsami.8b11527

Gong, S., Lai, D. T. H., Su, B., Si, K. J., Ma, Z., Yap, L. W., et al. (2015). Highly Stretchy Black Gold E-Skin Nanopatches as Highly Sensitive Wearable Biomedical Sensors. Adv. Electron. Mater. 1, 1400063. doi:10.1002/ aelm.201400063

Gong, S., Schwalb, W., Wang, Y., Chen, Y., Tang, Y., Si, J., et al. (2014). A Wearable and Highly Sensitive Pressure Sensor with Ultrathin Gold Nanowires. Nat. Commun. 5, 3132. doi:10.1038/ncomms4132

Green Marques, D., Alhais Lopes, P., T. de Almeida, A. A., Majidi, C., and Tavakoli, M. (2019). Reliable Interfaces for EGaIn Multi-Layer Stretchable Circuits and Microelectronics. Lab. Chip 19, 897-906. doi:10.1039/c8lc01093e

Han, S., Liu, C., Xu, H., Yao, D., Yan, K., Zheng, H., et al. (2018). Multiscale Nanowire-Microfluidic Hybrid Strain Sensors with High Sensitivity and Stretchability. Npj Flex. Electron. 2, 16. doi:10.1038/s41528-018-0029-x

Hao, B., Mu, L., Ma, Q., Yang, S., and Ma, P.-C. (2018). Stretchable and Compressible Strain Sensor Based on Carbon Nanotube Foam/polymer Nanocomposites with Three-Dimensional Networks. Composites Sci. Technol. 163, 162-170. doi:10.1016/j.compscitech.2018.05.017

Jeong, Y. R., Park, H., Jin, S. W., Hong, S. Y., Lee, S.-S., and Ha, J. S. (2015). Highly Stretchable and Sensitive Strain Sensors Using Fragmentized Graphene Foam. Adv. Funct. Mater. 25, 4228-4236. doi:10.1002/adfm.201501000

Kato, H., Itagaki, N., and Im, H. J. (2019). Growth and Raman Spectroscopy of Thickness-Controlled Rotationally Faulted Multilayer Graphene. Carbon 141, 76-82. doi:10.1016/j.carbon.2018.09.017

Kim, K. S., Zhao, Y., Jang, H., Lee, S. Y., Kim, J. M., Kim, K. S., et al. (2009). Largescale Pattern Growth of Graphene Films for Stretchable Transparent Electrodes. Nature 457, 706-710. doi:10.1038/nature07719

Lee, J., Kim, S., Lee, J., Yang, D., Park, B. C., Ryu, S., et al. (2014). A Stretchable Strain Sensor Based on a Metal Nanoparticle Thin Film for Human Motion Detection. Nanoscale 6, 11932-11939. doi:10.1039/C4NR03295K 
Lee, S., Shi, Q., and Lee, C. (2019). From Flexible Electronics Technology in the Era of IoT and Artificial Intelligence toward Future Implanted Body Sensor Networks. APL Mater. 7, 031302. doi:10.1063/1.5063498

Li, C., Cui, Y.-L., Tian, G.-L., Shu, Y., Wang, X.-F., Tian, H., et al. (2015). Flexible CNT-Array Double Helices Strain Sensor with High Stretchability for Motion Capture. Sci. Rep. 5, 15554. doi:10.1038/srep15554

Li, Q., Li, J., Tran, D., Luo, C., Gao, Y., Yu, C., et al. (2017). Engineering of Carbon Nanotube/polydimethylsiloxane Nanocomposites with Enhanced Sensitivity for Wearable Motion Sensors. J. Mater. Chem. C 5, 11092-11099. doi: $10.1039 / \mathrm{c} 7 \mathrm{tc} 03434 \mathrm{~b}$

Li, T., Li, Y., and Zhang, T. (2019). Materials, Structures, and Functions for Flexible and Stretchable Biomimetic Sensors. Acc. Chem. Res. 52, 288-296. doi:10.1021/ acs.accounts.8b00497

Li, X., Zhang, R., Yu, W., Wang, K., Wei, J., Wu, D., et al. (2012). Stretchable and Highly Sensitive Graphene-On-Polymer Strain Sensors. Sci. Rep. 2, 870. doi:10.1038/srep00870

Liao, X., Zhang, Z., Liang, Q., Liao, Q., and Zhang, Y. (2017). Flexible, Cuttable, and Self-waterproof Bending Strain Sensors Using Microcracked Gold Nanofilms@ paper Substrate. ACS Appl. Mater. Inter. 9, 4151-4158. doi:10.1021/ acsami.6b12991

Liu, C., Han, S., Xu, H., Wu, J., and Liu, C. (2018). Multifunctional Highly Sensitive Multiscale Stretchable Strain Sensor Based on a Graphene/Glycerol-KCl Synergistic Conductive Network. ACS Appl. Mater. Inter. 10, 31716-31724. doi:10.1021/acsami.8b12674

Liu, Q., Chen, J., Li, Y., and Shi, G. (2016). High-performance Strain Sensors with Fish-scale-like Graphene-Sensing Layers for Full-Range Detection of Human Motions. ACS Nano 10, 7901-7906. doi:10.1021/acsnano.6b03813

Liu, R., Kuang, X., Deng, J., Wang, Y.-C., Wang, A. C., Ding, W., et al. (2018). Shape Memory Polymers for Body Motion Energy Harvesting and Self-Powered Mechanosensing. Adv. Mater. 30, 1705195. doi:10.1002/adma.201705195

Liu, Y., Zhang, D., Wang, K., Liu, Y., and Shang, Y. (2016). A Novel Strain Sensor Based on Graphene Composite Films with Layered Structure. Composites A: Appl. Sci. Manufacturing 80, 95-103. doi:10.1016/j.compositesa.2015.10.010

Pang, C., Lee, G.-Y., Kim, T.-i., Kim, S. M., Kim, H. N., Ahn, S.-H., et al. (2012). A Flexible and Highly Sensitive Strain-Gauge Sensor Using Reversible Interlocking of Nanofibres. Nat. Mater 11, 795-801. doi:10.1038/nmat3380

Park, C. W., Moon, Y. G., Seong, H., Jung, S. W., Oh, J.-Y., Na, B. S., et al. (2016). Photolithography-based Patterning of Liquid Metal Interconnects for Monolithically Integrated Stretchable Circuits. ACS Appl. Mater. Inter. 8, 15459-15465. doi:10.1021/acsami.6b01896

Park, K.-I., Son, J. H., Hwang, G.-T., Jeong, C. K., Ryu, J., Koo, M., et al. (2014). Highly-efficient, Flexible Piezoelectric PZT Thin Film Nanogenerator on Plastic Substrates. Adv. Mater. 26, 2514-2520. doi:10.1002/adma.201305659

Park, M., Im, J., Shin, M., Min, Y., Park, J., Cho, H., et al. (2012). Highly Stretchable Electric Circuits from a Composite Material of Silver Nanoparticles and Elastomeric Fibres. Nat. Nanotech 7, 803-809. doi:10.1038/nnano.2012.206

Park, S.-J., Kim, J., Chu, M., and Khine, M. (2016). Highly Flexible Wrinkled Carbon Nanotube Thin Film Strain Sensor to Monitor Human Movement. Adv. Mater. Technol. 1, 1600053. doi:10.1002/admt.201600053

Qin, Y., Peng, Q., Ding, Y., Lin, Z., Wang, C., Li, Y., et al. (2015). Lightweight, Superelastic, and Mechanically Flexible Graphene/polyimide Nanocomposite Foam for Strain Sensor Application. ACS Nano 9, 8933-8941. doi:10.1021/ acsnano.5b02781

Roh, E., Hwang, B.-U., Kim, D., Kim, B.-Y., and Lee, N.-E. (2015). Stretchable, Transparent, Ultrasensitive, and Patchable Strain Sensor for Human-Machine Interfaces Comprising a Nanohybrid of Carbon Nanotubes and Conductive Elastomers. ACS Nano 9, 6252-6261. doi:10.1021/acsnano.5b01613

Shin, U.-H., Jeong, D.-W., Park, S.-M., Kim, S.-H., Lee, H. W., and Kim, J.-M. (2014). Highly Stretchable Conductors and Piezocapacitive Strain Gauges Based on Simple Contact-Transfer Patterning of Carbon Nanotube Forests. Carbon 80, 396-404. doi:10.1016/j.carbon.2014.08.079
Song, K., Han, J. H., Lim, T., Kim, N., Shin, S., Kim, J., et al. (2016). Subdermal Flexible Solar Cell Arrays for Powering Medical Electronic Implants. Adv. Healthc. Mater. 5, 1572-1580. doi:10.1002/adhm.201600222

Tao, L.-Q., Wang, D.-Y., Tian, H., Ju, Z.-Y., Liu, Y., Pang, Y., et al. (2017). Selfadapted and Tunable Graphene Strain Sensors for Detecting Both Subtle and Large Human Motions. Nanoscale 9, 8266-8273. doi:10.1039/ c7nr01862b

Tian, Z., He, J., Chen, X., Wen, T., Zhai, C., Zhang, Z., et al. (2018). Core-shell Coaxially Structured Triboelectric Nanogenerator for Energy Harvesting and Motion Sensing. RSC Adv. 8 (6), 2950-2957. doi:10.1039/C7RA12739A

Wang, D.-Y., Tao, L.-Q., Liu, Y., Zhang, T.-Y., Pang, Y., Wang, Q., et al. (2016). High Performance Flexible Strain Sensor Based on Self-Locked Overlapping Graphene Sheets. Nanoscale 8, 20090-20095. doi:10.1039/C6NR07620C

Wang, S., Xu, J., Wang, W., Wang, G.-J. N., Rastak, R., Molina-Lopez, F., et al. (2018). Skin Electronics from Scalable Fabrication of an Intrinsically Stretchable Transistor Array. Nature 555, 83-88. doi:10.1038/nature25494

Wang, Y., Hao, J., Huang, Z., Zheng, G., Dai, K., Liu, C., et al. (2018). Flexible Electrically Resistive-type Strain Sensors Based on Reduced Graphene OxideDecorated Electrospun Polymer Fibrous Mats for Human Motion Monitoring. Carbon 126, 360-371. doi:10.1016/j.carbon.2017.10.034

Wang, Y., Wang, L., Yang, T., Li, X., Zang, X., Zhu, M., et al. (2014). Wearable and Highly Sensitive Graphene Strain Sensors for Human Motion Monitoring. Adv. Funct. Mater. 24, 4666-4670. doi:10.1002/adfm.201400379

Wu, J.-B., Lin, M.-L., Cong, X., Liu, H.-N., and Tan, P.-H. (2018). Raman Spectroscopy of Graphene-Based Materials and its Applications in Related Devices. Chem. Soc. Rev. 47, 1822-1873. doi:10.1039/c6cs00915h

Xu, M., Qi, J., Li, F., and Zhang, Y. (2018). Highly Stretchable Strain Sensors with Reduced Graphene Oxide Sensing Liquids for Wearable Electronics. Nanoscale 10, 5264-5271. doi:10.1039/c7nr09022f

Yan, C., Wang, J., Kang, W., Cui, M., Wang, X., Foo, C. Y., et al. (2014). Highly Stretchable Piezoresistive Graphene-Nanocellulose Nanopaper for Strain Sensors. Adv. Mater. 26, 2022-2027. doi:10.1002/adma.201304742

Yan, Y., Potts, M., Jiang, Z., and Sencadas, V. (2018). Synthesis of HighlyStretchable Graphene - Poly(glycerol Sebacate) Elastomeric Nanocomposites Piezoresistive Sensors for Human Motion Detection Applications. Composites Sci. Technol. 162, 14-22. doi:10.1016/j.compscitech.2018.04.010

Zhang, Q., Di, Y., Huard, C. M., Guo, L. J., Wei, J., and Guo, J. (2015). Highly Stable and Stretchable Graphene-Polymer Processed Silver Nanowires Hybrid Electrodes for Flexible Displays. J. Mater. Chem. C 3, 1528-1536. doi: $10.1039 / \mathrm{c} 4 \mathrm{tc} 02448 \mathrm{f}$

Conflict of Interest: YF, HZ, and HT were employed by the company GRINM Group Co., Ltd.

The remaining authors declare that the research was conducted in the absence of any commercial or financial relationships that could be construed as a potential conflict of interest.

Publisher's Note: All claims expressed in this article are solely those of the authors and do not necessarily represent those of their affiliated organizations, or those of the publisher, the editors, and the reviewers. Any product that may be evaluated in this article, or claim that may be made by its manufacturer, is not guaranteed or endorsed by the publisher.

Copyright $\odot 2022$ Fan, Zhao, Yang, Yang, Ren and Tu. This is an open-access article distributed under the terms of the Creative Commons Attribution License (CC BY). The use, distribution or reproduction in other forums is permitted, provided the original author(s) and the copyright owner(s) are credited and that the original publication in this journal is cited, in accordance with accepted academic practice. No use, distribution or reproduction is permitted which does not comply with these terms. 